\title{
Dot-Like Formation of Metal Nanocrystals from Exfoliated Ruthenate Nanosheets*
}

\author{
Katsutoshi Fukuda ${ }^{\dagger}$ \\ Office of Society-Academia Collaboration for Innovation, \\ Kyoto University, Gokasho Uji, Kyoto 611-0011, Japan \\ Kazuhiro Kumagai \\ Surface and Nano-Analysis Section, Nanomaterial Characterization Division, \\ National Metrology Institute of Japan, National Institute of Advanced Industrial Science and Technology, \\ Tsukuba Central 5, 1-1-1 Higashi, Tsukuba, Ibaraki 305-8565, Japan \\ (Received 5 December 2013; Accepted 4 February 2014; Published 8 March 2014)
}

\begin{abstract}
We studied the metallization behavior of molecularly thin $\mathrm{Ru}_{0.95} \mathrm{O}_{2}^{0.2-}$ nanosheets obtained from complete delamination of a layered ruthenate, $\mathrm{K}_{0.2} \mathrm{RuO}_{2.1}$. Heating at $200^{\circ} \mathrm{C}$ under a mixture of $\mathrm{N}_{2}$ and $\mathrm{H}_{2}$ transformed multilayers of the nanosheets into $h c p$-Ru metals. These metals have unique morphology reflecting the multilayer structure of the precursors. Interestingly, a monolayer state of the $\mathrm{Ru}_{0.95} \mathrm{O}_{2}^{0.2-}$ nanosheets triggered a dot-like formation of $\mathrm{Ru}$ metal nanocrystals about $0.6 \mathrm{~nm}$ thick and several tens of nanometers wide, which represent a new family of 2D anisotropic metal nanomaterials. [DOI: 10.1380/ejssnt.2014.97]
\end{abstract}

Keywords: Ru; Metallic films; Nano structure chemistry

\section{INTRODUCTION}

Atomically controlled metal nanomaterials such as nanoparticles, nanorods, and nanowires have been widely studied. As they display intriguing physical and chemical properties associated with their surface state and shape at the nanometer scale, it is of importance to investigate their unique structures and dimensions for applications including electronics, optics, and magnetic devices, as well as catalysis [1].

Exfoliation of layered compounds such as clays [2, 3], oxides [4, 5], and graphite oxides [6, 7] into elemental host layers, called "exfoliated nanosheets", is a cuttingedge technology that promises to allow two-dimensional (2D) control of nanomaterials. The nanosheets are a few nanometers thick and several micrometers wide. Thanks to their polyelectrolytic nature, they can be used as inorganic building blocks for electrostatic self-assembly based on oppositely charged ions, polymers, and molecules. Such artificial nano-architectures, i.e., atomically controlled reactants, cannot be easily attained by conventional solid-state reaction, and often give rise to unpredictable thermodynamic reactivity. For instance, heating monolayer films of lepidocrocite-related $\mathrm{Ti}_{0.91} \mathrm{O}_{2}^{0.36-}$ nanosheets yielded anatase nanocrystals with a $c$-axis orientation [8]. The high dependency of this crystallization behavior on the number of deposited nanosheet layers indicates that both nucleation and growth of the anatase from the nanosheet film require extensive thermal activation of atomic diffusion. In the last decade, the chemical reduction of exfoliated graphite oxide nanosheets [7] has been shown to give distinct graphitic structures such as single- or multi-layered graphene, which can be regarded as a series of atomically controlled carbon nanomateri-

\footnotetext{
*This paper was presented at the 12th International Conference on Atomically Controlled Surfaces, Interfaces and Nanostructures (ACSIN-12) in conjunction with the 21st International Colloquium on Scanning Probe Microscopy (ICSPM21), Tsukuba International Congress Center, Tsukuba, Japan, November 4-8, 2013.

†Corresponding author: k-fukuda@saci.kyoto-u.ac.jp
}

als. Thus, secondary conversions of exfoliated nanosheets could pave the way to the creation of novel anisotropic nanomaterials.

More recently, we demonstrated that heating at up to $200^{\circ} \mathrm{C}$ under a mixture of inert gas and hydrogen caused topotactic conversion of a monolayer film of $2 \mathrm{D}$ hexagonal-type $\mathrm{RuO}_{2}^{0.2-}$ nanosheets [9] obtained via exfoliation of $\mathrm{NaRuO}_{2}$ into $\mathrm{Ru}$ metal nanosheets [10]. This unusual metallization can be understood as a lowtemperature activation sufficient to release $\mathrm{O}$ atoms from the oxide nanosheets without extensive thermal diffusion of the constituent $\mathrm{Ru}$ atoms. The structural similarity between the $2 \mathrm{D}$ hexagonal precursor nanosheets with $a=0.29 \mathrm{~nm}$ and the resultant metal nanosheets with $a=0.27 \mathrm{~nm}$ seems to play an important role in the metallization, and motivated us to investigate the effect of initial structure on the metallization behavior. Here, we report that reduction of $2 \mathrm{D}$ oblique-type $\mathrm{Ru}_{0.95} \mathrm{O}_{2}^{0.2-}$ nanosheets obtained from the delamination of $\mathrm{K}_{0.2} \mathrm{RuO}_{2.1}[11,12]$ triggered the dot-like formation of $2 \mathrm{D}$ anisotropic $\mathrm{Ru}$ metal nanocrystals.

\section{EXPERIMENTAL}

\section{A. Material Synthesis}

A dark-brown suspension of exfoliated ruthenate nanosheets was prepared as reported [12]. In brief, a pelleted mixture of $\mathrm{K}_{2} \mathrm{CO}_{3}$ and $\mathrm{RuO}_{2}$ rutile (molar ratio of 5:8) was heated at $850^{\circ} \mathrm{C}$ for $12 \mathrm{~h}$ under $\mathrm{Ar}$ flow. The obtained sample was ground in an agate mortar and washed with ultrapure water $(>18 \mathrm{M} \Omega)$, yielding a layered potassium ruthenate, $\mathrm{K}_{0.2} \mathrm{RuO}_{2.1} \cdot n \mathrm{H}_{2} \mathrm{O}(n=0-1)$. Ion-exchange of the interlayer potassium was conducted with $1 \mathrm{~mol} \mathrm{dm}^{-3} \mathrm{HCl}$ for 3 days at $60^{\circ} \mathrm{C}$, resulting in layered protonic ruthenate, $\mathrm{H}_{0.2} \mathrm{RuO}_{2.1} \cdot n \mathrm{H}_{2} \mathrm{O}(n=0$ $0.9)$ [12]. Then $0.4 \mathrm{~g}$ of this layered protonic ruthenate was added to $100 \mathrm{~cm}^{3}$ of tetrabutylammonium hydroxide (TBA-OH) aqueous solution. The molar ratio of TBA ions to the exchangeable protons in the ruthenate precursor was 10:1. The mixture was vigorously shaken 
for more than 10 days to exfoliate the layered ruthenate into elemental host layers. Non-exfoliated ruthenate was removed by subsequent centrifugation at $2000 \mathrm{rpm}$ for $30 \mathrm{~min}$. The resultant suspension of unilamellar nanosheets with a chemical composition of $\mathrm{Ru}_{0.95} \mathrm{O}_{2}^{0.2-}$ was used for monolayer film fabrication.

\section{B. Monolayer Film fabrication}

The ruthenate nanosheets were closely adsorbed on flat substrates via electrostatic self-assembly [13]. First, Si wafer with a native oxide and $\mathrm{SiO}_{2}$ glass substrates were

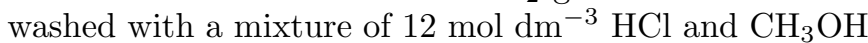
(1:1 by volume), followed by $18 \mathrm{~mol} \mathrm{dm}{ }^{-3} \mathrm{H}_{2} \mathrm{SO}_{4}$ and finally ultrapure water. The substrates were then immersed for $10 \mathrm{~min}$ in an aqueous solution of 1 mass\% cationic diblock copolymer composed of $\sim 14 \%$ polyvinylamine and $\sim 86 \%$ polyvinylalcohol to form a positively charged surface, and then reacted with the suspension containing anionic $\mathrm{Ru}_{0.95} \mathrm{O}_{2}^{0.2-}$ nanosheets $\left(0.08 \mathrm{~g} \mathrm{dm}^{-3}\right)$ for $20 \mathrm{~min}$. After dipping, the film sample was carefully drawn out and washed with ultrapure water to remove physically adsorbed species. Heat-treatments of these films were performed by increasing the temperature from ambient temperature at a rate of $5^{\circ} \mathrm{C} \mathrm{min}^{-1}$ to a preset temperature $\left(200,300,400,500^{\circ} \mathrm{C}\right)$ under gas-flow-controlled condition $\left(10 \% \mathrm{H}_{2}\right.$ and $\left.90 \% \mathrm{~N}_{2}, 250 \mathrm{ml} \mathrm{min}^{-1}\right)$. After keeping the samples at that temperature for 1 hour, they were cooled naturally in a tube furnace.

\section{Measurements and analysis}

Powder x-ray diffraction (XRD) patterns were collected by means of a Bragg-Brentano-type diffractometer (Rigaku Rint 2000) with $\mathrm{Cu} \mathrm{K} \alpha$ radiation $(\lambda=$ $0.15405 \mathrm{~nm}$ ). Mass loss up to $500^{\circ} \mathrm{C}$ under a mixture of $10 \% \mathrm{H}_{2}$ and $90 \% \mathrm{~N}_{2}$ was measured by thermogravimetry (TG) (Rigaku Thermo Plus TG 8120). The microscopic structure of the layered ruthenate and the restacked film of nanosheets after the reduction treatment under $10 \%$ $\mathrm{H}_{2}+90 \% \mathrm{~N}_{2}$ at $200^{\circ} \mathrm{C}$ was characterized by low-voltage scanning electron microscope (LV-SEM; Omicron NanoTechnology NanoSAM Lab with UHV-Gemini) [14]. All specimens were observed without a conductive coating under a primary electron beam of $700 \mathrm{eV}$ to reveal the true surface morphology. A frequency modulation atomic force microscope (FM-AFM; JEOL JSPM-5400) with a 7-nmdiameter Si-tip cantilever $\left(42 \mathrm{~N} \mathrm{~m}^{-1}\right)$ was used to obtain a high-resolution image of the morphological features of the nanosheets on the Si substrate. In-plane diffraction data for the as-grown nanosheet film and its heated derivatives were acquired by four-axis diffractometer equipped with a $\mathrm{NaI}$ scintillation counter in BL6C of the Photon Factory at the High Energy Accelerator Research Organization, Tsukuba, Japan.

\section{RESULTS AND DISCUSSION}

First, we considered the reduction of bulk-layered ruthenate, $\mathrm{H}_{0.2} \mathrm{RuO}_{2.1} \cdot 0.7 \mathrm{H}_{2} \mathrm{O}$, consisting of regularly



FIG. 1: XRD patterns for metallized products obtained from (a) a powder of the protonic layered precursor and (b) a restacked film of the $\mathrm{Ru}_{0.95} \mathrm{O}_{2}^{0.2-}$ nanosheets. Insets show surface-sensitive LV-SEM images.

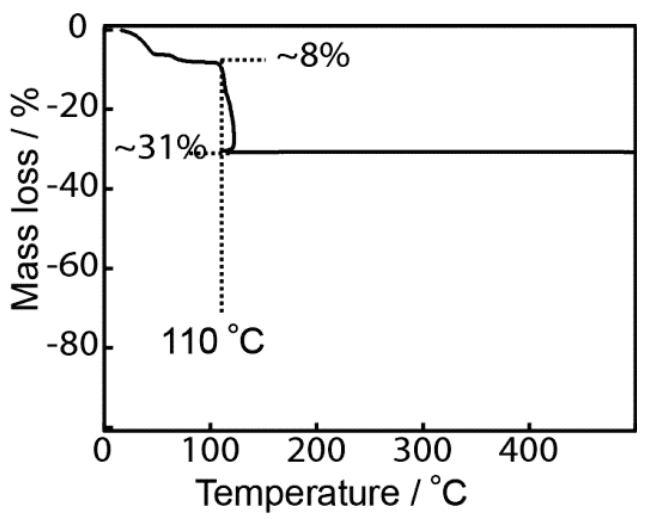

FIG. 2: A TG curve of layered ruthenate, $\mathrm{H}_{0.2} \mathrm{RuO}_{2.1} \cdot 0.7 \mathrm{H}_{2} \mathrm{O}$, used as a precursor for the $\mathrm{Ru}_{0.95} \mathrm{O}_{2}^{0.2-}$ nanosheets, under a flow of $90 \% \mathrm{~N}_{2}$ and $10 \% \mathrm{H}_{2}$.

stacked slabs of $\mathrm{RuO}_{6}$ octahedra, interlayer protons, and water [12]. Heating this material under a flow of $10 \%$ $\mathrm{H}_{2}$ and $90 \% \mathrm{~N}_{2}$ at $200^{\circ} \mathrm{C}$ yielded a powder sample with a metallic luster. The peaks in the powder XRD pattern after the heating were attributable to a single phase of bulk $\mathrm{Ru}$ metal, suggesting that the layered ruthenate was reduced (Fig. 1(a)). LV-SEM observation of the Ru metal powder revealed layered morphology in which the individual layers have many defects (inset). Mass loss of the protonated precursor up to $500^{\circ} \mathrm{C}$ revealed that metallization began at $110^{\circ} \mathrm{C}$ (Fig. 2). Mass loss of rutile-type $\mathrm{RuO}_{2}$ nanoparticles occurred at a comparably low temperature [15]. The mass loss of approximately $31 \%$ matches well with the $31.5 \%$ mass difference between 


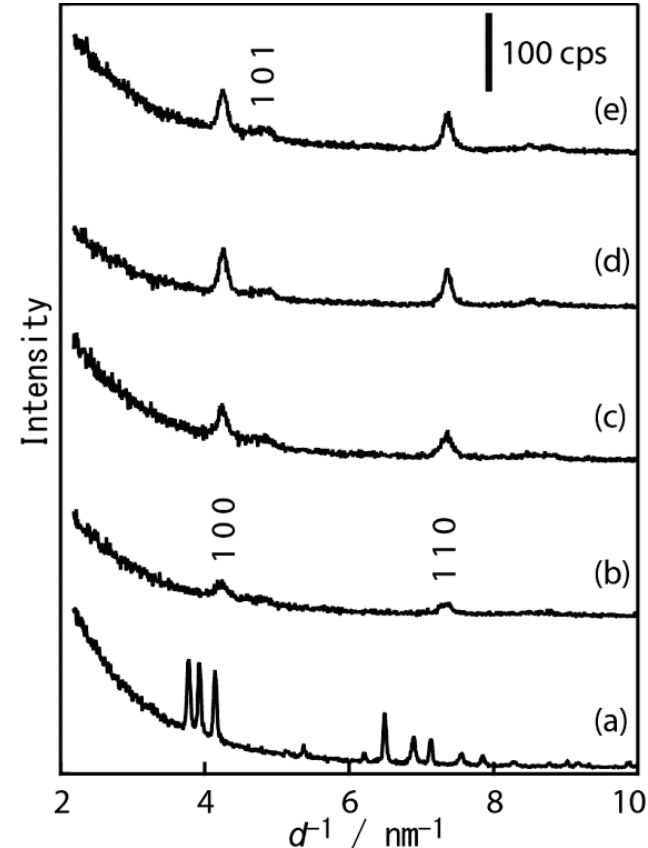

FIG. 3: In-plane XRD patterns for (a) as-grown monolayer film, and film heated at (b) 200, (c) 300, (d) 400, and (e) $500^{\circ} \mathrm{C}$.

$\mathrm{H}_{0.2} \mathrm{RuO}_{2.1} \cdot 0.7 \mathrm{H}_{2} \mathrm{O}$ and $\mathrm{Ru}$ metal, indicating complete metallization.

Next, to investigate metallization behavior in the multilayer state, we obtained a thin film composed of restacked $\mathrm{Ru}_{0.95} \mathrm{O}_{2}^{0.2-}$ nanosheets by drying a droplet of the exfoliated nanosheet suspension on the $\mathrm{SiO}_{2}$ substrate. XRD analysis of the film (not shown) showed a series of strong diffraction peaks typical of lamellar ordering with $d=$ $1.68 \mathrm{~nm}[11,12]$. This value can be explained by a layered structure in which the $\mathrm{Ru}_{0.95} \mathrm{O}_{2}^{0.2-}$ nanosheets interleaved with TBA ions. After treatment under $10 \% \mathrm{H}_{2}+90 \% \mathrm{~N}_{2}$ at $200^{\circ} \mathrm{C}$, a metallic film exhibiting only one diffraction peak assignable to 002 of $\mathrm{Ru}$ metal in a halo pattern derived from the substrate was formed (Fig. 1(b)). This peak indicates that the $\mathrm{Ru}$ film has a strong $c$-axis orientation perpendicular to the substrate, despite the use of the amorphous surface. An LV-SEM image (inset) of a cross-section of the $\mathrm{Ru}$ metal physically peeled from the substrate shows that the lamellar morphology of the bulk case is roughly preserved.

The above two cases suggest the transformation of the ruthenate system into layered $\mathrm{Ru}$ metals with preferential orientation. In other words, the $2 \mathrm{D}$ anisotropy of the $\mathrm{RuO}_{6}$ slab in the $\mathrm{Ru}_{0.95} \mathrm{O}_{2}^{0.2-}$ nanosheet should affect the nucleation and growth of the Ru metal. The defects observed in these morphological changes should account for the difference in $\mathrm{Ru}$ atom density between the precursor nanosheet and the produced $\mathrm{Ru}$ metal.

To identify the transition in an extremely thin $\mathrm{Ru}_{0.95} \mathrm{O}_{2}^{0.2-}$ nanosheet in a monolayer state, we used a monolayer film in which the $\mathrm{Ru}_{0.95} \mathrm{O}_{2}^{0.2-}$ nanosheets lay flat to the $\mathrm{SiO}_{2}$ glass substrate. An in-plane XRD pattern of the as-deposited monolayer film showed a lot of diffraction peaks indexed by a $2 \mathrm{D}$ oblique cell with


FIG. 4: AFM images of the $\mathrm{Ru}_{0.95} \mathrm{O}_{2}^{0.2-}$ nanosheets in a $2 \mathrm{D}$ oblique lattice (a) before and (b, c) after metallization. Broken circle in (c) indicates dot-like formation of Ru metal nanocrystals. Inset in (c) is a height profile along the line $\mathrm{X}-\mathrm{Y}$.

$a=0.561(1) \mathrm{nm}, b=512(1) \mathrm{nm}$, and $\gamma=109.4(2)^{\circ}$ (Fig. 3(a)) [12]. After reduction at $200^{\circ} \mathrm{C}$, only two XRD peaks were observed (Fig. 3(b)). As judged from the metallization behavior of the bulk systems mentioned earlier, they can be indexed as 100 and 110 of $h c p-\mathrm{Ru}$. A lack of indexes associated with the $00 l$ direction in the in-plane direction can be explained by the formation of $\mathrm{Ru}$ metal with a $c$-axis orientation perpendicular to the substrate. This preferential growth is consistent with the case of the multilayer state. As the reduction temperature was increased, a small peak at $4.7 \mathrm{~nm}^{-1}$ appeared (Figs. 3(c)(e)). This peak is assignable to 101 of $\mathrm{Ru}$ metal, which is the strongest reflection in the polycrystalline $\mathrm{Ru}$ metal. Heat treatment above $300^{\circ} \mathrm{C}$ induces a rearrangement of the $\mathrm{Ru}$ atoms via thermal diffusion, consequently leading to a collapse of the $c$-axis orientation.

We used a true noncontact FM-AFM to examine morphological changes before and after the metallization of the monolayer film on a flat $\mathrm{Si}$ substrate. The asdeposited $\mathrm{Ru}_{0.95} \mathrm{O}_{2}^{0.2-}$ nanosheets appear as lamellar objects with an average thickness of $1.3 \pm 0.1 \mathrm{~nm}$ and a width ranging from sub-micrometer to micrometer size (Fig. 4(a)). Their crystallographic thickness cannot be 
defined accurately at present, as the precise crystal structure of the parent layered potassium ruthenate has still not been resolved. However, combined with the $d$-spacing of $0.456 \mathrm{~nm}$ revealed by XRD analysis of the anhydrous layered protonic ruthenate [12], the observed thickness of the $\mathrm{Ru}_{0.95} \mathrm{O}_{2}^{0.2-}$ nanosheets in the AFM image is strong evidence of a single layer of $\mathrm{RuO}_{6}$ octahedra, as is the case with the $\mathrm{RuO}_{2}^{0.2-}$ nanosheets obtained from $\mathrm{NaRuO}_{2}$. The same AFM technique revealed that the $\mathrm{RuO}_{2}^{0.2-}$ nanosheets, being composed of a single layer of edge-shared $\mathrm{RuO}_{6}$ octahedra ( 0.4-0.5 nm thickness), had an average thickness of about $1.3 \mathrm{~nm}$ [9]. This apparently larger thickness measured by AFM than the crystallographic thickness can be explained by the presence of adsorbed species, possibly water molecules and charge-compensating protons, on the surface of the anionic nanosheets [16]. A majority of the $\mathrm{Ru}_{0.95} \mathrm{O}_{2}^{0.2-}$ nanosheets were adsorbed as a monolayer on the substrate, although some gaps and overlaps between the nanosheets were inevitable under the fabrication recipe used. After the metallization, spotty objects were observed (Fig. 4(b)). High-resolution imagery revealed a dot-like formation of Ru metal nanocrystals from the oxide nanosheets (Fig. 4(c)). Interestingly, their average height of $\sim 0.6 \mathrm{~nm}$ is much smaller than those of the precursor oxide nanosheets and conventional metal nanoparticles. Given the diameter of the tip used in this measurement, the nanocrystals seem to have a lateral size of several tens of nanometers. A coverage analysis based on height histograms of Figs. 4(a) and 4(b) (not shown) shows a decrement in the coverage from approximately $95 \%$ to less than $85 \%$ as a result of the gathering of the $\mathrm{Ru}$ ions to produce the $\mathrm{Ru}$ metal nanocrystals. Such shrinkage in both thickness and width likely stems from a large difference between the precursor nanosheets and the nanocrystals in the density of the $\mathrm{Ru}$ atoms in the in-plane direction.

Unlike the $\mathrm{Ru}_{0.95} \mathrm{O}_{2}^{0.2-}$ nanosheets, the $\mathrm{RuO}_{2}^{0.2-}$ nanosheets [9] gave rise to a topotactic metallization, i.e. transformation from "nanosheet" to "nanosheet", despite the same reduction conditions. The resultant $\mathrm{Ru}$ metal nanosheets consist of $\mathrm{Ru}$ atoms in a $2 \mathrm{D}$ hexagonal package [10]. Shrinkage of their sheet size as estimated from AFM analyses agrees fairly well with a decrement of the in-plane lattice from $0.29 \mathrm{~nm}$ to $0.27 \mathrm{~nm}$, about $7 \%$. This is due to a similar atomic arrangement of the $\mathrm{Ru}$ atoms in these materials with the $2 \mathrm{D}$ hexagonal periodic structure. In practice, the $\mathrm{Ru}$ metal nanosheets produced early in the heating process $\left(<200^{\circ} \mathrm{C}\right)$ have a slightly larger $a$-axis than that of bulk Ru metal. This fact may be evidence that the oxide nanosheets work as an embryo. Further heating at $>300^{\circ} \mathrm{C}$ caused a structural relaxation: the $a$-axis of the $\mathrm{Ru}$ metal nanosheets became nearly the same as that of bulk Ru metal. Also, the growth of polycrystalline $\mathrm{Ru}$ metals was promoted until the polycrystalline component became dominant. On the other hand, the peak position of 100 and 110 of the $\mathrm{Ru}$ metal nanocrystals obtained here remained unchanged up to $500^{\circ} \mathrm{C}$. The polycrystalline phase is still a minor component in the monolayer film heated at $500^{\circ} \mathrm{C}$. Once the $\mathrm{Ru}$ metal nanocrystals are generated owing to the thermal diffusion of the constituent $\mathrm{Ru}$ atoms in the $\mathrm{Ru}_{0.95} \mathrm{O}_{2}^{0.2-}$ nanosheet, their isolated environment needs more intense thermal diffusion to grow as well. Thus, the dotlike $\mathrm{Ru}$ metal nanocrystals formed from the $\mathrm{Ru}_{0.95} \mathrm{O}_{2}^{0.2-}$ nanosheet should arise from the atomic-scale mismatch that requires a local gathering of the $\mathrm{Ru}$ atoms for nucleation. Nevertheless, the Ru metal nanocrystals have a similar thickness to that of the $\mathrm{Ru}$ metal nanosheets obtained via the topotactic conversion. A single layer of $\mathrm{Ru}$ crystals with various lateral sizes would be produced. Our findings demonstrate the great potential of atomic-scale reactants composed of exfoliated nanosheets as a solutionbased nanotechnology for controlling the 2D anisotropy of metal nanocrystals.

\section{CONCLUSIONS}

Our results show that reduction of the $\mathrm{Ru}_{0.95} \mathrm{O}_{2}^{0.2-}$ nanosheets led to the formation of thin metal nanocrystals presumably because of the large mismatch in the lateral arrangement of the $\mathrm{Ru}$ atoms. As the initial inplane structure of the precursor nanosheets governs the resulting metal morphology, this would be of significant interest not only for fundamental metal sciences, but also for applications for the synthesis of conductive or catalytic materials. The unique metallization dependent on nanostructured reactants may pave the way for creating a diversity of anisotropic metal nanomaterials, including nanosheets and nanodots.

\section{Acknowledgments}

We thank Prof. W. Sugimoto (Shinshu Univ.), Prof. T. Sasaki (NIMS), and Prof. T. Sekiguchi (NIMS) for their liberal cooperation. This work was partly supported by a Grant-in-Aid for Young Scientists (B) of JSPS, 22750186 and 24710130. The in-plane XRD measurements were performed with the approval of the Photon Factory Program Advisory Committee (2011G003, 2013G033).
[1] C. S. S. R. Kumar (ed.), Metallic Nanomaterials (WileyVCH Verlag, Weinheim, 2009).

[2] G. F. Walker, Nature 187, 312 (1960).

[3] P. H. Nadeau, M. J. Wilson, W. J. McHardy, and J. M. Tait, Science 225, 923 (1984).

[4] M. M. Treacy, S. B. Rice, A. J. Jacobson, and J. T. Lewandowski, Chem. Mater. 2, 279 (1990).

[5] T. Sasaki, M. Watanabe, H. Hashizume, H. Yamada, and
H. Nakazawa, J. Am. Chem. Soc. 118, 8329 (1996).

[6] K. S. Novoselov, A. K. Geim, S. V. Morozov, D. Jiang, Y. Zhang, S. V. Dubonos, I. V. Grigorieva, and A. A. Firsov, Science 306, 666 (2004).

[7] S. Stankovich, D. A. Dikin, G. H. B. Dommett, K. M. Kohlhaas, E. J. Zimney, E. A. Stach, R. D. Piner, S. T. Nguyen, and R. S. Ruoff, Nature 442, 282 (2006).

[8] K. Fukuda, Y. Ebina, T. Shibata, T. Aizawa, I. Nakai, 
and T. Sasaki, J. Am. Chem. Soc. 129, 202 (2007).

[9] K. Fukuda, T. Saida, J. Sato, M. Yonezawa, Y. Takasu, and W. Sugimoto, Inorg. Chem. 49, 4391 (2010).

[10] K. Fukuda, J. Sato, T. Saida, W. Sugimoto, Y. Ebina, T. Shibata, M. Osada, and T. Sasaki, Inorg. Chem. 52, 2280 (2013).

[11] W. Sugimoto, H. Iwata, Y. Yasunaga, Y. Murakami, and Y. Takasu, Angew. Chem. Int. Ed. 42, 4092 (2003).

[12] K. Fukuda, H. Kato, J. Sato, W. Sugimoto, and Y. Takasu, J. Solid State Chem. 182, 2997 (2009).
[13] J. Sato, H. Kato, M. Kimura, K. Fukuda, and W. Sugimoto, Langmuir 26, 18049 (2010).

[14] K. Kumagai, T. Sekiguchi, K. Fukuda, and T. Sasaki, Appl. Phys. Express 2, 105504 (2009).

[15] M. Prudenziati, B. Morten, and E. Travan, Mater. Sci. Engineer. B 98, 167 (2003).

[16] T. Sasaki, Y. Ebina, Y. Kitami, M. Watanabe, and T. Oikawa, J. Phys. Chem. B 105, 6116 (2001). 